\title{
Understanding professional autonomy: Nurse initiated application
}

\section{Gagnon $L^{*}$ \\ School of Nursing, Laurentian University, Ontario, Canada}

The purpose of this perspective article is to disseminate knowledge on an important issue in the nursing workplace, such as the concept of autonomy that has been identified and explored in the literature. In the ethical literature, autonomy has been defined on three levels namely physiological, personal, and social. Conversely, in the nursing literature, autonomy has also been discussed in three contexts specifically as a rights-based autonomy, individual occupation-related autonomy, and professional autonomy.

Rights-based autonomy can be expanded in the context of patients, whereas occupation-related autonomy refers to the work context of nurses, and professional autonomy focuses on the discipline of nursing. These three contexts of autonomy reflect discourse in the nursing literature and relate to either practitioners or recipients of care. The contexts make a difference in understanding the significance of the concept of autonomy in healthcare.

The nursing literature subsequently identifies defining attributes of autonomy such as independence, capacity for decision-making, judgment, knowledge, and self-determination. These attributes involve active freedom of will, and the ability to make choices based on scientific, personal and experiential knowledge, thus yielding conscious decisions. These identified attributes apply to a rights-based notion of autonomy, occupation-related autonomy, and professional autonomy.

Copyright: (C)2020 Gagnon L. This is an open-access article distributed under the terms of the Creative Commons Attribution License, which permits unrestricted use, distribution, and reproduction in any medium, provided the original author and source are credited.
Professional autonomy is further clarified in the following scenario. A certified oncology nurse with specialty knowledge and skills caring for this patient population can demonstrate autonomy's defining attributes. For example, the nurse works with a patient to begin the oncology treatment. The nurse helps the patient navigate the cancer care system and coordinates the treatment and supportive care. The nurse is responsible for monitoring the patient's response to the treatment and the cancer experience. In this situation, the nurse uses a nursing framework to guide patient assessment and independently uses his/her specialized knowledge and clinical judgment to recognize real or potential problems related to coping with the treatment and the disease, and to assess the need for further support. These actions show nurse-initiated treatments thus reflecting professional autonomy in nursing interventions and activities.

In refining the defining attributes of autonomy, it is beneficial to identify probabilities about the concept such as formal education, gained experience, self-discipline, exposure to a system of beliefs, and acceptance of responsibility. As a result, there are consequences of autonomy such as individual professional status, freedom for authority and power, and accountability.

Given its various contexts, understanding autonomy can help inform nursing theory and practice. Subsequently, these reflections can aid in shaping the future of healthcare. 